ARTICLE

Received 13 Feb 2013 | Accepted 11 Sep 2013 | Published 28 Oct 2013

DOI: $10.1038 /$ ncomms 3594

OPEN

\title{
Quantum mechanical which-way experiment with an internal degree of freedom
}

Konrad Banaszek ${ }^{1}$ Paweł Horodecki²,3, Michał Karpiński ${ }^{1, \dagger} \&$ Czesław Radzewicz $^{1}$

For a particle travelling through an interferometer, the trade-off between the available whichway information and the interference visibility provides a lucid manifestation of the quantum mechanical wave-particle duality. Here we analyse this relation for a particle possessing an internal degree of freedom such as spin. We quantify the trade-off with a general inequality that paints an unexpectedly intricate picture of wave-particle duality when internal states are involved. Strikingly, in some instances which-way information becomes erased by introducing classical uncertainty in the internal degree of freedom. Furthermore, even imperfect interference visibility measured for a suitable set of spin preparations can be sufficient to infer absence of which-way information. General results are illustrated with a proof-of-principle single-photon experiment.

\footnotetext{
${ }^{1}$ Faculty of Physics, University of Warsaw, ul. Hoża 69, PL-00-681 Warszawa, Poland. ${ }^{2}$ Faculty of Applied Physics and Mathematics, Technical University of Gdańsk, ul. Narutowicza 11/12, PL-80-952 Gdańsk, Poland. ${ }^{3}$ National Quantum Information Center of Gdańsk, ul. Wł. Andersa 27, PL-81-824 Sopot, Poland. $\dagger$ Present address: Clarendon Laboratory, University of Oxford, Parks Road, Oxford OX1 3PU, UK. Correspondence and requests for materials should be addressed to K.B. (email: Konrad.Banaszek@fuw.edu.pl).
} 
$\mathrm{T}$ he duality between wave and particle properties of a microscopic physical system is a founding principle of quantum mechanics ${ }^{1,2}$. A canonical illustration is provided by a single particle travelling through a double slit or a MachZehnder interferometer: an attempt to gain information about the path taken by the particle inevitably reduces the visibility of the interference pattern ${ }^{3-11}$. The purpose of this work is to analyse the trade-off between interference visibility and which-way information for a particle equipped with an internal degree of freedom, for example, spin. In such a general case, the interaction with an environment that acquires which-way information can transform in a non-trivial manner the joint path-spin state of the particle. This opens up an interesting question of how to infer the amount of which-way information deposited in the environment from visibility measurements. Another issue is, to what extent manipulating the spin subsystem can control the information about the path taken by the particle.

A common approach to quantify the amount of which-way information is to use distinguishability $D$, defined as the maximum difference between the probabilities of correct and incorrect identification $^{12}$ of the path taken by the particle inside the interferometer, based on the state of the environment. We assume here that both paths are equiprobable. In the spinless case, the ability for the particle distributed between two paths to interfere is characterized by the visibility $V$, which measures the modulation depth of interference fringes after the paths are combined at the interferometer exit. These two quantities are related by the inequality ${ }^{6}$

$$
D^{2}+V^{2} \leq 1
$$

When the particle travelling through the interferometer has an internal structure, the amount of available which-way information depends in principle on the preparation of the spin subsystem and the specifics of the interaction with the environment. Here we demonstrate that in this case the strongest bound on the distinguishability $D$ is obtained by replacing $V$ with a quantity named generalized visibility that depends on the initial spin preparation and the effective quantum channel experienced by the particle resulting from the interaction with the environment. Further, we present a systematic method to construct estimates for the generalized visibility based on directly measurable quantities, that is, interference visibilities for particular spin preparations at the input and selections of spin states before combining the paths at the interferometer exit. This provides an efficient strategy to find an upper bound on the available whichway information without performing full quantum process tomography ${ }^{13-15}$. We illustrate the general results with a proofof-principle single-photon experiment that also demonstrates how which-way information can be erased by introducing mixedness in the spin preparation.

\section{Results}

The inequality. The quantum mechanical system considered here is a particle present in one of two distinguishable paths, denoted respectively as $|0\rangle_{Q}$ and $|1\rangle_{Q}$. In addition, the particle is equipped with a $d$-dimensional internal degree of freedom - spin - which we will treat as a subsystem $S$. The complete state of the particle prepared in the interferometer is characterized by a certain density operator $\hat{\varrho}_{Q S}$. Both paths are equiprobable, that is, $\operatorname{Tr}_{S}\left({ }_{Q}\left\langle 0\left|\hat{\varrho}_{Q S}\right| 0\right\rangle_{Q}\right)=\operatorname{Tr}_{S}\left({ }_{Q}\left\langle 1\left|\hat{\varrho}_{Q S}\right| 1\right\rangle_{Q}\right)=1 / 2$. Inside the interferometer, the particle interacts with an environment $E$ producing a joint state $\varrho_{Q S E}^{\prime}$. The interaction is constrained in that it cannot transfer the particle between the paths. In this setting, which-way information contained in the environment corresponds to the ability to discriminate between two normalized density matrices $\hat{\varrho}_{E}^{\prime(i)}=2 \operatorname{Tr}_{S}\left({ }_{Q}\left\langle i\left|\varrho_{Q}^{\prime}{ }_{Q S E}\right| i\right\rangle_{Q}\right), i=0$, 1 . In the general case of mixed states, distinguishability is given explicitly by the expression ${ }^{12,16}$

$$
D=\frac{1}{2}\left\|\hat{\varrho}_{E}^{\prime(0)}-\hat{\varrho}_{E}^{\prime(1)}\right\|,
$$

where $\|\cdot\|$ denotes the trace norm. Let us stress here that this quantity is not conditioned upon any measurement performed eventually on subsystems QS.

Our aim is to infer an upper bound on $D$ from the properties of the particle, that is, subsystems $Q S$, after the interaction with the environment. The state of the particle at this stage can be written as a result of an action of a certain quantum channel ${ }^{17} \boldsymbol{\Lambda}$ on the input state $\hat{\varrho}_{Q S}$, with $\boldsymbol{\Lambda}\left(\hat{\varrho}_{Q S}\right)=\operatorname{Tr}_{E}\left(\hat{\varrho}_{Q S E}^{\prime}\right)$. Because the particle is not transferred between interferometer arms, for any spin operator $\hat{\sigma}_{S}$, we have

$$
\boldsymbol{\Lambda}\left(|i\rangle_{Q}\langle j| \otimes \hat{\sigma}_{S}\right)=|i\rangle_{Q}\langle j| \otimes \boldsymbol{\Lambda}_{i j}\left(\hat{\sigma}_{S}\right), \quad i, j=0,1 .
$$

Our central result is that $D$ satisfies the inequality:

$$
D^{2}+V_{G}^{2} \leq 1,
$$

where $V_{G}$, named generalized visibility, reads:

$$
V_{G}=d\left\|\left(\mathbf{I} \otimes \boldsymbol{\Lambda}_{01}\right)\left(\left(\hat{\mathbb{1}} \otimes \sqrt{\hat{\varrho}_{0}}\right)\left|\Phi_{+}\right\rangle\left\langle\Phi_{+}\right|\left(\hat{\mathbb{1}} \otimes \sqrt{\hat{\varrho}_{1}}\right)\right)\right\| .
$$

Here $\left|\Phi_{+}\right\rangle$is a normalized maximally entangled state of two replicas of the spin subsystem, $\mathbf{I}$ is the identity channel, $\hat{\mathbb{1}}$ is the identity operator on the spin subsystem and $\hat{\varrho}_{i}=2_{Q}\left\langle i\left|\hat{\varrho}_{Q S}\right| i\right\rangle_{Q}$ are normalized spin states for the particle in either arm of the interferometer before the interaction with the environment. The complete proof of the inequality is presented in Methods.

Examples. The inequality (4) resembles that for a spinless particle given in equation (1), but the concept of generalized visibility covers a much wider range of physical scenarios. In order to illustrate its breadth, let us consider here three cases. First, when no interaction with the environment occurs, $\boldsymbol{\Lambda}_{01}=\mathbf{I}$. In this case $V_{G}=\sqrt{\operatorname{Tr} \hat{\varrho}_{0} \operatorname{Tr} \hat{\varrho}_{1}}=1$, that is, no matter what internal states for individual paths are initially chosen, the environment does not gain any which-way information.

Second, let us take $\Lambda_{01}(\hat{\sigma})=\hat{\sigma}_{0} \operatorname{Tr} \hat{\sigma}$, where $\hat{\sigma}_{0}$ is a fixed unittrace hermitian operator. Physically, this occurs when the internal degree of freedom is entirely transferred to the environment and replaced with a state $\hat{\sigma}_{0}$. Then $V_{G}=\left\|\sqrt{\hat{\varrho}_{0}} \sqrt{\hat{\varrho}_{1}}\right\|$ which is the standard expression for quantum fidelity ${ }^{18,19}$ between density operators $\hat{\varrho}_{0}$ and $\hat{\varrho}_{1}$. Consequently, the only way to ensure that no which-way information leaks out is to associate both the interferometer paths with identical internal states.

Finally, suppose that

$$
\boldsymbol{\Lambda}_{01}(\hat{\sigma})=\frac{1}{d} \hat{\sigma}^{T}
$$

where $T$ denotes transposition. This channel gives $V_{G}=$ $\frac{1}{d}\left\|\sqrt{\hat{\varrho}_{0}}\right\|\left\|\sqrt{\hat{\varrho}_{1}}\right\|$. The resulting expression has non-intuitive properties: for a particle prepared in any pure state $\left(|0\rangle_{Q}\left|\psi_{0}\right\rangle_{S}+|1\rangle_{Q}\left|\psi_{1}\right\rangle_{S}\right) / \sqrt{2}$ the generalized visibility equals $1 / d$, which is strictly less than one for a non-trivial spin subsystem when $d \geq 2$. Generalized visibility reaches one only when the two paths are associated with maximally mixed spin states, $\hat{\varrho}_{0}=\hat{\varrho}_{1}=\frac{1}{d} \hat{1}$, which follows immediately from the inequality between arithmetic and quadratic means. This indicates that which-way information deposited in the environment may be erased by introducing classical noise in the input state of the spin subsystem. To illuminate this point, let us take a single photon travelling through a Mach-Zehnder interferometer and consider its polarization, spanned by two orthogonal states $|h\rangle_{S}$ and $|v\rangle_{S}$, as the two-dimensional internal degree of freedom. Let the 
interaction of the photon with the environment be given by a unitary map

$$
\begin{aligned}
& \frac{1}{\sqrt{2}}\left(|0\rangle_{Q}\left|\psi_{0}\right\rangle_{S}+|1\rangle_{Q}\left|\psi_{1}\right\rangle_{S}\right)\left|e_{1}\right\rangle_{E} \rightarrow \frac{1}{2}\left[| 0 \rangle _ { Q } \left(\psi_{0 h}|h\rangle_{S}\left|e_{1}\right\rangle_{E}\right.\right. \\
& \left.\quad+\psi_{0 h}|v\rangle_{S}\left|e_{2}\right\rangle_{E}+\psi_{0 v}|h\rangle_{S}\left|e_{3}\right\rangle_{E}+\psi_{0 v}|v\rangle_{S}\left|e_{4}\right\rangle_{E}\right) \\
& \quad+|1\rangle_{Q}\left(\psi_{1 h}|h\rangle_{S}\left|e_{1}\right\rangle_{E}+\psi_{1 v}|h\rangle_{S}\left|e_{2}\right\rangle_{E}\right. \\
& \left.\left.\quad+\psi_{1 h}|v\rangle_{S}\left|e_{3}\right\rangle_{E}+\psi_{1 v}|v\rangle_{S}\left|e_{4}\right\rangle_{E}\right)\right]
\end{aligned}
$$

where $\left|e_{n}\right\rangle_{E}, \quad n=1, \ldots, 4$, are four orthogonal states of the environment and $\psi_{i h}={ }_{s}\left\langle h \mid \psi_{i}\right\rangle_{S}, \quad \psi_{i v}={ }_{s}\left\langle v \mid \psi_{i}\right\rangle_{s}$ are the probability amplitudes in the rectilinear basis. It is easy to verify that this map indeed induces $\Lambda_{01}(\hat{\sigma})=\hat{\sigma}^{T} / 2$, although the polarization state in an individual arm is scrambled to a completely mixed state, $\boldsymbol{\Lambda}_{00}(\hat{\sigma})=\boldsymbol{\Lambda}_{11}(\hat{\sigma})=\operatorname{Tr}(\hat{\sigma}) \hat{1} / 2$.

If we prepare $\left|\psi_{0}\right\rangle_{S}=\left|\psi_{1}\right\rangle_{S}=|h\rangle_{S}$, the two states of the environment correlated with the paths

$$
\begin{aligned}
& \hat{\varrho}_{E}^{\prime(0)}=\frac{1}{2}\left(\left|e_{1}\right\rangle_{E}\left\langle e_{1}|+| e_{2}\right\rangle_{E}\left\langle e_{2}\right|\right), \\
& \hat{\varrho}_{E}^{\prime(1)}=\frac{1}{2}\left(\left|e_{1}\right\rangle_{E}\left\langle e_{1}|+| e_{3}\right\rangle_{E}\left\langle e_{3}\right|\right),
\end{aligned}
$$

are partly distinguishable. Similarly, for the vertical input polarization, $\left|\psi_{0}\right\rangle_{S}=\left|\psi_{1}\right\rangle_{S}=|v\rangle_{S}$, the environment states are

$$
\begin{aligned}
& \hat{\varrho}_{E}^{\prime}(0)=\frac{1}{2}\left(\left|e_{3}\right\rangle_{E}\left\langle e_{3}|+| e_{4}\right\rangle_{E}\left\langle e_{4}\right|\right), \\
& \hat{\varrho}_{E}^{\prime}(1)=\frac{1}{2}\left(\left|e_{2}\right\rangle_{E}\left\langle e_{2}|+| e_{4}\right\rangle_{E}\left\langle e_{4}\right|\right) .
\end{aligned}
$$

However, if the input polarization is prepared in a completely mixed state $\frac{1}{2}\left(|h\rangle_{S}\langle h|+| v\rangle_{S}\langle v|\right)$, one sees immediately that both the paths are associated with the same mixed environment state $(1 / 4) \sum_{k=1}^{4}\left|e_{k}\right\rangle_{E}\left\langle e_{k}\right|$ and which-way information is not available anymore. The inequality (4) demonstrates that this effect is an intrinsic feature of the channel under consideration rather than its specific realization given in equation (7).

Estimating generalized visibility. Generalized visibility $V_{G}$ is given by a rather intricate expression involving the spin preparation and the effective quantum channel experienced by the particle. In principle, full information about the channel can be obtained from quantum process tomography ${ }^{13-15}$, but this approach may be resource consuming, especially for a high dimension of the internal subsystem $S$. We will now give a recipe how to construct estimates for $V_{G}$ from direct visibility measurements for specific spin preparations and selections of individual spin components before interfering the particle paths.

Consider the following procedure. The particle is prepared in one of states

$$
\left|\psi^{\mu}\right\rangle_{Q S}=\frac{1}{\sqrt{2}}\left(|0\rangle_{Q}\left|\psi_{0}^{\mu}\right\rangle_{S}+|1\rangle_{Q}\left|\psi_{1}^{\mu}\right\rangle_{S}\right)
$$

where the normalized kets $\left|\psi_{0}^{\mu}\right\rangle_{S}$ and $\left|\psi_{1}^{\mu}\right\rangle_{S}$ describe the spin state in the upper and lower interferometer path. The index $\mu$ labels different preparations. After the interaction with the environment, the spin subsystem is filtered individually in each interferometer arm to extract probability amplitudes corresponding to normalized spin states $\left|\chi_{0}^{v}\right\rangle_{S}$ and $\left|\chi_{1}^{v}\right\rangle_{S}$. These components are subsequently made indistinguishable by a suitable spin transformation and interfered on a balanced beam splitter with a relative phase shift $\phi$. The probabilities of detecting the particle at the two output ports \pm of the beam splitter read

$$
p_{ \pm}^{\mu v}(\phi)=\frac{1}{2}\left[p^{\mu v} \pm \operatorname{Re}\left(V^{\mu v} e^{i \phi}\right)\right] .
$$

Here,

$$
p^{\mu v}=\frac{1}{2}\left[\left\langle\chi_{0}^{v}\left|\boldsymbol{\Lambda}_{00}\left(\left|\psi_{0}^{\mu}\right\rangle\left\langle\psi_{0}^{\mu}\right|\right)\right| \chi_{0}^{v}\right\rangle+\left\langle\chi_{1}^{v}\left|\boldsymbol{\Lambda}_{11}\left(\left|\psi_{1}^{\mu}\right\rangle\left\langle\psi_{1}^{\mu}\right|\right)\right| \chi_{1}^{v}\right\rangle\right]
$$

is the overall probability of detecting the particle in the filtered components. The modulation depth of interference fringes is characterized by the fractional visibility, given explicitly by

$$
V^{\mu v}=\left\langle\chi_{0}^{v}\left|\boldsymbol{\Lambda}_{01}\left(\left|\psi_{0}^{\mu}\right\rangle\left\langle\psi_{1}^{\mu}\right|\right)\right| \chi_{1}^{v}\right\rangle .
$$

For the quantities introduced here, we always have $\left|V^{\mu v}\right| \leq p^{\mu v}$. Full-depth modulation of fringes is observed for the equality sign.

Let us now take any set of complex coefficients $\alpha_{\mu v}$ such that a linear combination of rank-one operators $\left(\left|\psi_{0}^{\mu}\right\rangle\left\langle\psi_{1}^{\mu}\right|\right)^{T} \otimes$ $\left|\chi_{1}^{v}\right\rangle\left\langle\chi_{0}^{v}\right|$ can be written as

$$
\begin{array}{r}
\sum_{\mu \nu} \alpha_{\mu v}\left(\left|\psi_{0}^{\mu}\right\rangle\left\langle\psi_{1}^{\mu}\right|\right)^{T} \otimes\left|\chi_{1}^{v}\right\rangle\left\langle\chi_{0}^{v}\right| \\
=\left(\sqrt{\hat{\varrho}_{1}^{T}} \otimes \hat{\mathbb{1}}\right) \hat{U}\left(\sqrt{\hat{\varrho}_{0}^{T}} \otimes \hat{1}\right)
\end{array}
$$

for a certain operator $\hat{U}$ acting on the duplicated spin subsystem that satisfies $\hat{U}^{\dagger} \hat{U} \leq \hat{\mathbb{1}} \otimes \hat{\mathbb{1}}$. We show in Methods that under these assumptions the generalized visibility is bounded by a linear combination of fractional visibilities with the same coeffcients $\alpha_{\mu v}$ :

$$
V_{G} \geq\left|\sum_{\mu v} \alpha_{\mu v} V^{\mu v}\right| \text {. }
$$

Combining this result with equation (4) yields a family of bounds on distinguishability in the form

$$
D \leq \sqrt{1-V_{G}^{2}} \leq \sqrt{1-\left|\sum_{\mu \nu} \alpha_{\mu \nu} V^{\mu \nu}\right|^{2}} .
$$

Special cases of equation (16) are intuitive. Consider a scenario shown in Fig. 1. We use only a single preparation $\left(|0\rangle_{Q}\right.$ $\left.\left|\psi_{0}\right\rangle_{S}+|1\rangle_{Q}\left|\psi_{1}\right\rangle_{S}\right) / \sqrt{2}$ (henceforth in this example we drop the superfluous label $\mu$ ), and the sets of filter states in the upper $\left\{\left|\chi_{0}^{v}\right\rangle_{S}\right\}$ and lower $\left\{\left|\chi_{1}^{v}\right\rangle_{S}\right\}$ arms form two orthonormal bases for the spin subsystem with $v$ indexing the basis elements. In equation (14), we can take arbitrary phase factors as coefficients $\alpha_{v}=e^{i \theta_{v}}$, with the operator $\hat{U}_{j}=\left(\left|\psi_{0}\right\rangle\left\langle\psi_{1}\right|\right)^{T} \otimes \sum_{v} e^{i \theta_{v}}$ $\left|\chi_{1}^{v}\right\rangle\left\langle\chi_{0}^{v}\right|$ satisfying the condition $\hat{U}^{\dagger} \hat{U} \leq \hat{\mathbb{1}} \otimes \hat{\mathbb{1}}$. According to equation (15), we have $V_{G} \geq\left|\sum_{v} e^{i \theta_{v}} V^{v}\right|$, which maximized over phases $\theta_{v}$ estimates the generalized visibility by a sum of absolute values of fractional visibilities:

$$
V_{G} \geq \sum_{v}\left|V^{v}\right|
$$

If for each filter we measure the maximum possible fractional visibility $\left|V^{v}\right|=p^{v}$, the right-hand side of equation (17) becomes one, because completeness of the bases $\left\{\left|\chi_{0}^{v}\right\rangle_{S}\right\}$ and $\left\{\left|\chi_{1}^{v}\right\rangle_{S}\right\}$ implies that $\sum_{v} p^{v}=1$. In other words, when the interaction with the environment introduces different phase shifts between distinguishable components of the spin subsystem but maintains overall coherence between the paths, we can observe full visibility by suitably sorting spin components at the output and interfering them pairwise. This suffices to verify that no which-way information is deposited in the environment. However, as noted earlier, the interplay between the path and the spin subsystems can exhibit more intricate behaviour. We illustrate this point with a single-photon experiment.

Experiment. To expose more complex aspects of the trade-off between which-way information and interference visibility for a particle with an internal structure, we investigated experimentally 


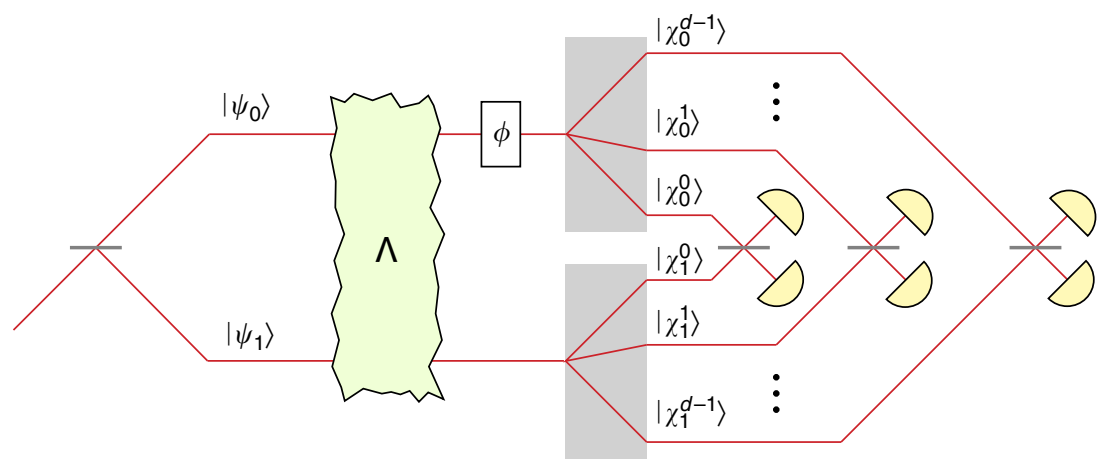

Figure 1 | Projective measurement of fractional visibilities. A particle is prepared in the spin state $\left|\psi_{0}\right\rangle$ in the upper arm and $\left|\psi_{1}\right\rangle$ in the lower arm. After interaction with the environment described by the channel $\boldsymbol{\Lambda}$ the phase between interferometer arms is modulated by $\phi$. Next, the spin states are filtered in the orthonormal bases $\left\{\left|\chi_{0}^{v}\right\rangle\right\}$ for the upper arm and $\left\{\left|\chi_{1}^{v}\right\rangle\right\}$ for the lower arm, and individual components are interfered pairwise to determine fractional visibilities $V^{v}, v=0,1, \ldots, d-1$.

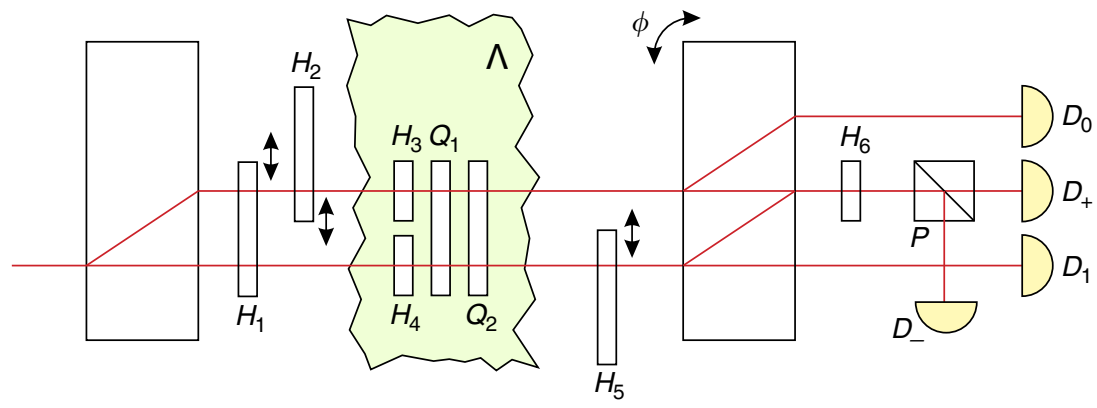

Figure 2 | Noisy Mach-Zehnder interferometer. Experimental set-up. A heralded single photon with $810 \mathrm{~nm}$ central wavelength and $3 \mathrm{~nm}$ bandwidth from a down-conversion source is split between two paths using a calcite crystal. Polarizations in individual paths are prepared using optional half-wave plates (HWPs) $H_{1}$ and $H_{2}$. Noise $\boldsymbol{\Lambda}$ is introduced using four sets of orientations of HWPs $H_{3}$ and $H_{4}$ and quarter-wave plates $Q_{1}$ and $Q_{2}$ mounted on computer-controlled motorized rotation stages. Polarization components of the output state are filtered using a HWP $\mathrm{H}_{5}$ and a second calcite crystal. Interference is realized in the common-path configuration using HWP $\mathrm{H}_{6}$ oriented at $45^{\circ}$ with respect to the set-up plane and a polarizing beam splitter $P$ with output ports monitored by single-photon detectors $D_{+}$and $D_{-}$. Two other detectors $D_{0}$ and $D_{1}$ are used to determine the overall count rate. The phase shift $\phi$ is introduced by rotating the second calcite crystal about an axis perpendicular to the set-up plane using a closed loop piezo actuator.

interference of a single photon in a noisy Mach-Zehnder interferometer shown in Fig. 2, using photon polarization as the internal subsystem $S$. Single photons with $810 \mathrm{~nm}$ central wavelength were generated by type-II spontaneous parametric down-conversion in a 30-mm long periodically poled potassium titanyl phosphate crystal pumped with $14 \mathrm{~mW}$ of $405 \mathrm{~nm}$ wavelength light from continuous wave diode laser, and heralded by detection of orthogonally polarized conjugate photons from the same pairs. The photons, after sending through a $3 \mathrm{~nm}$ bandwidth interference filter and transmitting through a single-mode fibre, were split between two paths using a calcite displacer. Equal splitting was ensured by using a fibre polarization controller. Removable half-wave plates $H_{1}$ and $H_{2}$ were used to prepare any combination of horizontal $h$ and vertical $v$ polarization states for the two paths.

Inside the interferometer the photons were subjected to path-dependent unitaries $|0\rangle_{Q}\left\langle 0\left|\otimes \hat{K}_{S}^{(0)}+\right| 1\right\rangle_{Q}\langle 1| \otimes \hat{K}_{S}^{(1)}$, where the pair $\left(\hat{K}_{S}^{(0)}, \hat{K}_{S}^{(1)}\right)$ was an equally weighted mixture of $(\hat{1}, \hat{\mathbb{1}}),(\hat{X}, \hat{X}),(\hat{Y},-\hat{Y})$ and $(\hat{Z}, \hat{Z})$, with $\hat{X}, \hat{Y}$ and $\hat{Z}$ denoting Pauli operators. The unitaries were realized by wave plates $H_{3}, H_{4}$, $Q_{1}, Q_{2}$ mounted on motorized rotation stages, with orientations specified in Table 1. This procedure scrambled the polarization state in a single arm to the completely mixed state. But the noise had a non-trivial effect on the joint path-polarization state, as

$$
\boldsymbol{\Lambda}_{01}(\hat{\sigma})=\frac{1}{4}(\hat{\sigma}+\hat{X} \hat{\sigma} \hat{X}-\hat{Y} \hat{\sigma} \hat{Y}+\hat{Z} \hat{\sigma} \hat{Z})=\frac{1}{2} \hat{\sigma}^{T}
$$

which provides a physical realization of equation (6) for $d=2$. We treat the realized noisy channel as a black box simulating interaction with an environment and analyse the maximum amount of which-way information that might have leaked to the environment in the worst-case scenario. We have seen that another realization of this channel, given in equation (7), can reveal certain which-way information depending on the preparation of the internal degree of freedom.

In order to estimate which-way information possibly deposited in the environment, we used four different combinations of preparations for the photon polarization in the upper and the lower paths, denoted jointly as $\mu=h h, h v, v h, v v$. After the simulated interaction with the environment, we filtered out from each path either horizontal or vertical component using the half-wave plate $\mathrm{H}_{5}$, which directed selected polarizations to the same output port after the second calcite crystal. We used a common-path set-up based on the half-wave plate $H_{6}$ and the polarizer $P$ monitored by single-photon detectors $D \pm$ to measure the corresponding fractional visibilities $V^{\mu v}$. The index $v$ labelling filters also assumes one of four values: $h h, h v, v h$ or $v v$. The two non-interfering output ports after the second calcite crystal were monitored by detectors $D_{0}$ and $D_{1}$ in order to normalize overall count rates. The measured count rates were adjusted by binomial resampling to correct for non-uniform detection efficiencies of the detectors.

Because individual paths inside the interferometer are completely depolarized by the interaction with the environment, 
Table 1 | Orientations of principal axes of wave plates used to simulate interaction with the environment.

\begin{tabular}{lcccr}
$\left(\hat{\boldsymbol{K}}_{\mathbf{S}}^{(\mathbf{0})}, \hat{\boldsymbol{K}}_{\mathbf{s}}^{(\mathbf{1})}\right)$ & $\mathbf{H}_{\mathbf{3}}$ & $\mathbf{H}_{\mathbf{4}}$ & $\mathbf{Q}_{\mathbf{1}}$ & \multicolumn{1}{c}{$\mathbf{Q}_{\mathbf{2}}$} \\
\hline$(\hat{1}, \hat{1})$ & $-45^{\circ}$ & $-45^{\circ}$ & $45^{\circ}$ & $45^{\circ}$ \\
$(\hat{X}, \hat{X})$ & $45^{\circ}$ & $45^{\circ}$ & $45^{\circ}$ & $-45^{\circ}$ \\
$(\hat{Y},-\hat{Y})$ & $0^{\circ}$ & $90^{\circ}$ & $45^{\circ}$ & $45^{\circ}$ \\
$(\hat{Z}, \hat{Z})$ & $0^{\circ}$ & $0^{\circ}$ & $45^{\circ}$ & $-45^{\circ}$ \\
\hline
\end{tabular}

the theoretical probability of filtering out the photon for any selection of the preparation $\mu$ and the filter $v$ equals $p^{\mu v}=1 / 2$. Theoretical predictions for fractional visibilities are collected in Table 2. It is seen that only few combinations of preparations and filters are expected to produce interference fringes. Let us select for a moment a single preparation $\mu$. After filtering a pair of polarization components, one could use the remaining components to measure also the second fractional visibility for complementary polarizations. For example, $v=v v$ is complementary to $v=h h$, and $v=v h$ is complementary to $v=h v$. This is an example of a scenario covered by the inequality (17). Although the photon is equally likely to choose either of the two complementary filters, interference fringes are visible only for one of them and the right-hand side of equation (17) is at most $1 / 2$.

Suppose now that before interaction with the environment the photon polarization is averaged to the completely mixed state and $\hat{\varrho}_{0}=\hat{\varrho}_{1}=(1 / 2) \hat{1}$, for example by switching randomly between four preparations $h h, h v, v h, v v$. To estimate which-way information available in this case, let us take on the left-hand side of equation (14) four non-zero coefficients:

$$
\begin{aligned}
& \alpha_{h h, h h}=\frac{1}{2} e^{i \theta_{1}}, \quad \alpha_{h v, v h}=\frac{1}{2} e^{i \theta_{2}}, \\
& \alpha_{v h, h v}=\frac{1}{2} e^{i \theta_{3}}, \quad \alpha_{v v, v v}=\frac{1}{2} e^{i \theta_{4}}
\end{aligned}
$$

where $\theta_{1}, \ldots, \theta_{4}$ are arbitrary phases. The corresponding operator

$$
\begin{aligned}
\hat{U}= & e^{i \theta_{1}}|h\rangle\langle h|\otimes| h\rangle\left\langle h\left|+e^{i \theta_{2}}\right| h\right\rangle\langle v|\otimes| v\rangle\langle h| \\
& +e^{i \theta_{3}}|v\rangle\langle h|\otimes| h\rangle\left\langle v\left|+e^{i \theta_{4}}\right| v\right\rangle\langle v|\otimes| v\rangle\langle v|
\end{aligned}
$$

is unitary for any choice of phases $\theta_{1}, \ldots, \theta_{4}$. Therefore, equation (15) provides a bound on generalized visibility in the form $V_{G} \geq\left|e^{i \theta_{1}} V^{h h, h h}+e^{i \theta_{2}} V^{h v, v h}+e^{i \theta_{3}} V^{v h, h v}+e^{i \theta_{4}} V^{v v, v v}\right| / 2$. Maximization over phases yields

$$
V_{G} \geq \frac{1}{2}\left(\left|V^{h h, h h}\right|+\left|V^{h v, v h}\right|+\left|V^{v h, h v}\right|+\left|V^{v v, v v}\right|\right) .
$$

This bound on generalized visibility can be applied directly to experimental data in order to estimate available which-way information for mixed polarization preparation.

In order to measure fractional visibilities, the phase in the interferometer was adjusted by rotating one of the calcite crystals about an axis perpendicular to the plane of the set-up using a closed loop piezo-electric actuator. For each selected phase value,
Table 3 | Experimental values of filtering probabilities $p^{\mu \nu}$ and fractional visibilities $V^{\mu \nu}$.

\begin{tabular}{llllll}
$\boldsymbol{\mu \nu}$ & $\boldsymbol{p}^{\boldsymbol{\mu \nu}}$ & $\boldsymbol{V}^{\boldsymbol{\mu} \boldsymbol{\nu}}$ & $\boldsymbol{\mu \nu}$ & $\boldsymbol{p}^{\boldsymbol{\mu \nu}}$ & $\boldsymbol{V}^{\boldsymbol{\mu \nu}}$ \\
\hline$h h, h h$ & 0.489 & 0.476 & $h h, v v$ & 0.512 & 0.104 \\
$h v, v h$ & 0.513 & 0.488 & $h v, h v$ & 0.490 & 0.039 \\
$v h, h v$ & 0.511 & 0.479 & $v h, v h$ & 0.490 & 0.032 \\
$v v, v v$ & 0.489 & 0.478 & $v v, h h$ & 0.512 & 0.100 \\
\hline \multicolumn{4}{l}{ Uncertainties are below 0.003 for all values shown. } \\
\hline
\end{tabular}
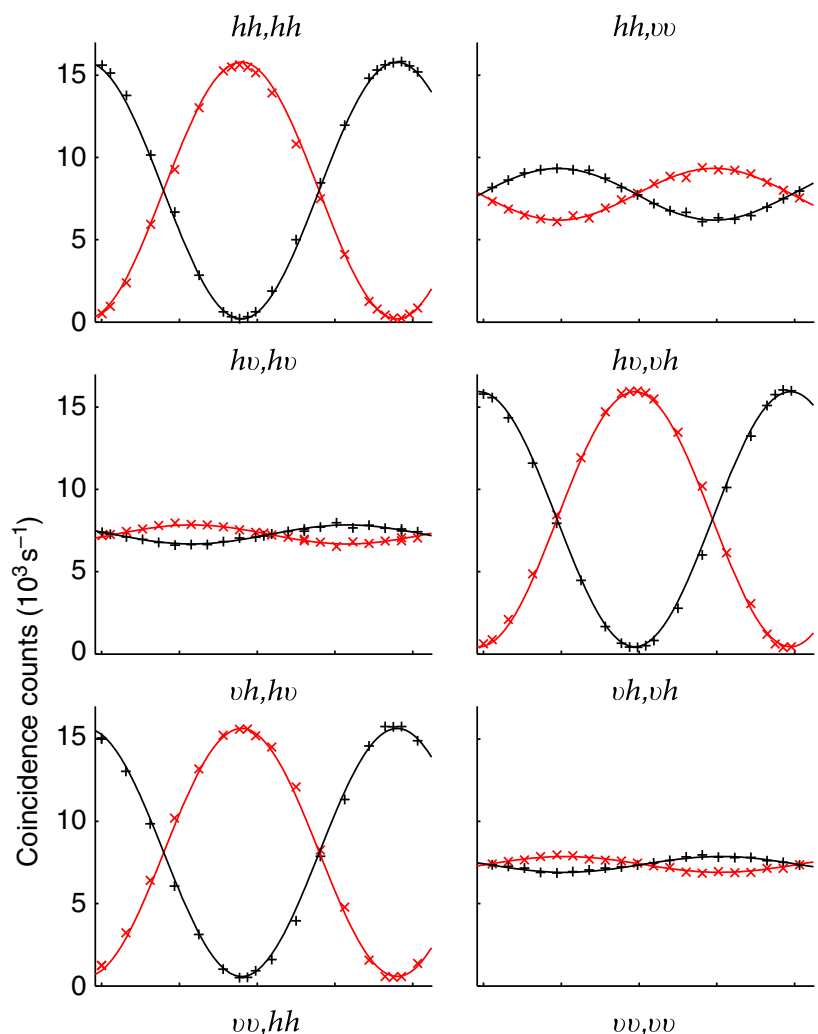

vh,vh
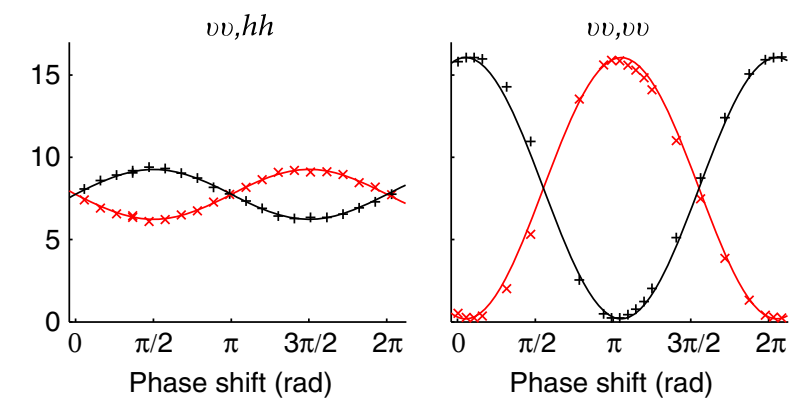

Figure 3 | Interference fringes in a noisy interferometer. Count rates on detectors $D_{+}(+$, black $)$and $D_{-}(x$, red $)$ conditioned upon detection of conjugate heralding photons from the down-conversion source as a function of the phase shift in the interferometer. Panels are labelled as $\mu, v$, where $\mu$ specifies polarization preparation and $v$ defines polarizations filtered from the two paths. Solid lines are sinusoidal fits used to determine fractional visibilities. Experimental uncertainties of count rates are smaller than the size of the symbols.

we averaged over four unitaries simulating the interaction with the environment. Experimentally measured interference fringes for combinations of preparations $\mu$ and filters $v$ entering the inequality (21) are shown in Fig. 3 along with results obtained for 
complementary filters. Probabilities $p^{\mu v}$ and fractional visibilities $V^{\mu \nu}$ determined from these measurements are collected in Table 3. Inserting these data in equation (21) gives $V_{G} \geq 0.960 \pm 0.006$. This translates into an upper bound on distinguishability in the form $D \leq 0.28 \pm 0.02$ according to equation (16). Remarkably, this stringent bound is determined from just few measurements of fractional visibilities rather than complete quantum process tomography. In contrast, consider an individual pure preparation and complementary projective filters, corresponding to a pair of graphs shown in a single row of Fig. 3. In this case, experimental results yield at most $V_{G} \geq 0.580 \pm 0.006$ using equation (17), which implies $D \leq 0.815 \pm 0.005$. This separation between estimated on distinguishability for mixed and pure polarization preparations strikingly demonstrates the convoluted effects of internal degrees of freedom in a which-way experiment.

\section{Discussion}

The scenario considered in this work assumed a clear distinction between the environment and the spin that are both external to the path subsystem. The amount of available which-way information was determined solely from the quantum state of the environment after the interaction with the particle in the interferometer and no classical information about either spin preparation or measurement results could be used for that purpose. This makes our approach distinct from previous studies of the quantum erasure phenomenon ${ }^{8,20-23}$, where postselection carried out on the environment can restore conditional interference fringes. Here, the environment was treated as an adversary whose information about the path taken by the particle we try to control and estimate by manipulating the spin subsystem at the preparation and the detection stages.

In this context, the analysis of a which-way experiment with an internal degree of freedom points at non-trivial issues in prepareand-measure protocols for quantum key distribution over complex noisy channels. Suppose that a cryptographic key is to be established by sending a particle along a randomly selected one of two paths, and the key security is to be verified by preparing occasionally the particle in a superposition of the two paths and measuring its coherence at the output by sampling interference fringes. If the particle is equipped with spin, the trade-off between distinguishability and visibility derived in this work implies that certain eavesdropping strategies require the sender to use randomness in spin preparation in order to ensure security. Further, to verify the security, the sender and the receiver may need to combine data collected for an array of spin preparations and filterings.

An entanglement-based analogue of the scenario analysed above would be to prepare jointly two particles in a maximally entangled path-spin state and to subject one of them to interaction with the environment modelling an eavesdropping attempt. In this case, spin can have the role of a shield subsystem fully protecting the privacy of a cryptographic key generated by detecting particles in individual paths, even though the noise present in the bipartite state may prevent entanglement distillation at the same rate as key generation ${ }^{24-27}$. To complete the parallel, estimates for generalized visibility determined from fractional visibility measurements can be viewed as a dynamical analogue of recently introduced privacy witnesses ${ }^{28}$.

\section{Methods}

Our basic tool will be the Choi-Jamiołkowski isomorphism ${ }^{29,30}$ between linear maps and operators acting on the duplicated Hilbert space. Let us define a maximally entangled state of two replicas of the system $Q S$ as $\left|\Phi_{+}\right\rangle_{Q S Q^{\prime} S^{\prime}}=\left|\Phi_{+}\right\rangle_{Q Q^{\prime}} \otimes\left|\Phi_{+}\right\rangle_{S S^{\prime}}, \quad$ where $\quad\left|\Phi_{+}\right\rangle_{Q Q^{\prime}}=\left(|0\rangle_{Q}|0\rangle_{Q^{\prime}}+|1\rangle_{Q}\right.$ $\left.|1\rangle_{Q^{\prime}}\right) / \sqrt{2}$ and $\left|\Phi_{+}\right\rangle_{S S^{\prime}}=\left(\sum_{l=0}^{d-1}|l\rangle_{S}|l\rangle_{S^{\prime}}\right) / \sqrt{d}$. The Choi-Jamiołkowski state corresponding to a channel $\boldsymbol{\Lambda}$ is given by $\hat{\Lambda}_{Q S Q^{\prime} S^{\prime}}=(\mathbf{I} \otimes \boldsymbol{\Lambda})\left(\left|\Phi_{+}\right\rangle_{Q S Q^{\prime} S^{\prime}}\left\langle\Phi_{+}\right|\right)$, where the identity map $\mathbf{I}$ acts on the subsystem $Q S$, whereas $\boldsymbol{\Lambda}$ acts on $Q^{\prime} S^{\prime}$. The transformation of a state $\hat{\varrho}^{\prime}=\boldsymbol{\Lambda}(\hat{\varrho})$ can be written as

$$
\hat{\varrho}_{Q^{\prime} S^{\prime}}^{\prime}=2 d \operatorname{Tr}_{Q S}\left[\hat{\Lambda}_{Q S Q^{\prime} S^{\prime}} \hat{\varrho}_{Q S}^{T}\right]
$$

where the transposition $T$ is performed in the same basis in which the state $\left|\Phi_{+}\right\rangle_{Q S Q^{\prime} S^{\prime}}$ has been defined. The input state $\hat{\varrho}$ is encoded in the subsystem $Q S$, whereas the output state is inscribed in the subsystem $Q^{\prime} S^{\prime}$

In order to take into account information deposited in the environment in course of the interaction, we will consider a purification of the state $\hat{\Lambda}_{Q S Q^{\prime} S^{\prime}}$ :

$$
\hat{\Lambda}_{Q S Q^{\prime} S^{\prime}}=\operatorname{Tr}_{E}\left(|\Lambda\rangle_{Q S Q^{\prime} S^{\prime} E}\langle\Lambda|\right) \text {. }
$$

Because the particle is not transferred between the two paths, the purified state has the form

$$
|\Lambda\rangle_{Q S Q^{\prime} S^{\prime} E}=\frac{1}{\sqrt{2}}\left(|00\rangle_{Q Q^{\prime}}\left|\Lambda_{0}\right\rangle_{S S^{\prime} E}+|11\rangle_{Q Q^{\prime}}\left|\Lambda_{1}\right\rangle_{S S^{\prime} E}\right),
$$

where the states $\left|\Lambda_{i}\right\rangle_{S S^{\prime} E}, i=0,1$, are normalized.

The joint state of the particle and the environment after the interaction can be written as follows:

$$
\begin{aligned}
\hat{\varrho}_{Q^{\prime} S^{\prime} E}^{\prime} & =2 d \operatorname{Tr}_{Q S}\left(|\Lambda\rangle_{Q S Q^{\prime} S^{\prime} E}\langle\Lambda| \hat{\varrho}_{Q S}^{T}\right) \\
& =\frac{d}{2} \sum_{i, j=0,1}|i\rangle_{Q^{\prime}}\langle j| \otimes \operatorname{Tr}_{S}\left[\left|\Lambda_{i}\right\rangle_{S^{\prime} E}\left\langle\Lambda_{j}\right|(2\langle i|\hat{\varrho}| j\rangle)_{S}^{T}\right]
\end{aligned}
$$

In the first expression, transposition is performed on subsystems QS, while in the second expression only on $S$. Thus, the environment states correlated with the particle present in one or another path are given after normalization by $\hat{\varrho}_{E}^{\prime}(i)=d \operatorname{Tr}_{S S^{\prime}}\left[\left|\Lambda_{i}\right\rangle_{S S^{\prime} E}\left\langle\Lambda_{i}\right|\left(\hat{\varrho}_{i}\right)_{S}^{T}\right]$, where $\hat{\varrho}_{i}=2_{Q}\left\langle i\left|\hat{\varrho}_{Q S}\right| i\right\rangle_{Q}$ are the normalized internal states of the particle in the upper and the lower arms of the interferometer. Distinguishability can only increase by access to additional subsystems that purify the states. We will take as purifications of $\hat{\varrho}_{E}^{\prime(i)}$ states $\left|\varepsilon_{i}\right\rangle_{S S^{\prime} E}=\hat{U}_{S S^{\prime}}^{(i)} \sqrt{d\left(\hat{\varrho}_{i}\right)_{S}^{T}}$ $\left|\Lambda_{i}\right\rangle_{S S^{\prime} E}$ where $\hat{U}_{S S^{\prime}}^{(i)}$ are arbitrary unitaries. For any choice of these unitaries, we will have

$$
D \leq \sqrt{1-\left.\left.\right|_{S S^{\prime} E}\left\langle\varepsilon_{0} \mid \varepsilon_{1}\right\rangle_{S S^{\prime} E}\right|^{2}} .
$$

The most stringent bound is obtained by minimizing the right-hand side over $\hat{U}_{S S^{\prime}}^{(0)}$ and $\hat{U}_{S S^{\prime}}^{(1)}$. This can be equivalently written as $D \leq \sqrt{1-V_{G}^{2}}$, where

$$
V_{G}=\max _{\hat{U}_{S S^{\prime}}}\left|S S^{\prime} E\left\langle\Lambda_{1}\left|\sqrt{d\left(\hat{\varrho}_{1}\right)_{S}^{T}} \hat{U}_{S S^{\prime}} \sqrt{d\left(\hat{\varrho}_{0}\right)_{S}^{T}}\right| \Lambda_{0}\right\rangle_{S S^{\prime} E}\right|
$$

and $\hat{U}_{S S^{\prime}}$ is an arbitrary unitary, or using the trace operation

$$
V_{G}=d \max _{\hat{U}_{S S^{\prime}}}\left|\operatorname{Tr}_{S S^{\prime}}\left(\hat{U}_{S S^{\prime}} \sqrt{\left(\hat{\varrho}_{0}\right)_{S}^{T}} \operatorname{Tr}_{E}\left(\left|\Lambda_{0}\right\rangle_{S S^{\prime} E}\left\langle\Lambda_{1}\right|\right) \sqrt{\left(\hat{\varrho}_{1}\right)_{S}^{T}}\right)\right| .
$$

The maximum can be expressed in terms of the trace norm as follows:

$$
V_{G}=d\left\|\sqrt{\left(\hat{\varrho}_{0}\right)_{S}^{T}} \operatorname{Tr}_{E}\left(\left|\Lambda_{0}\right\rangle_{S S^{\prime} E}\left\langle\Lambda_{1}\right|\right) \sqrt{\left(\varrho_{1}\right)_{S}^{T}}\right\| .
$$

The channel characteristics enters the above formula through

$$
\begin{aligned}
\operatorname{Tr}_{E}\left(\left|\Lambda_{0}\right\rangle_{S S^{\prime} E}\left\langle\Lambda_{1}\right|\right) & =2_{Q Q^{\prime}}\left\langle 00\left|\hat{\Lambda}_{Q S Q^{\prime} S^{\prime}}\right| 11\right\rangle_{Q Q^{\prime}} \\
& =\left(\mathbf{I} \otimes \boldsymbol{\Lambda}_{01}\right)\left(\left|\Phi_{+}\right\rangle_{S S^{\prime}}\left\langle\Phi_{+}\right|\right),
\end{aligned}
$$

where $\boldsymbol{\Lambda}_{01}$ has been defined in equation (3). Using the fact that $\left(\sqrt{\left(\hat{\varrho}_{i}\right)_{S}^{T}} \otimes \hat{\mathbb{1}}_{S^{\prime}}\right)$ $\left|\Phi_{+}\right\rangle_{S S^{\prime}}=\left(\hat{\mathbb{1}}_{S} \otimes \sqrt{\left(\hat{\varrho}_{i}\right)_{S^{\prime}}}\right)\left|\Phi_{+}\right\rangle_{S S^{\prime}}$ yields equation (5).

The expression for fractional visibilities $V^{\mu v}$ given in equation (13) can be rewritten in the tensor form

$$
V^{\mu v}=d \operatorname{Tr}\left\{\left[\left(\left|\psi_{0}^{\mu}\right\rangle_{S}\left\langle\psi_{1}^{\mu}\right|\right)^{T} \otimes\left|\chi_{1}^{v}\right\rangle_{S^{\prime}}\left\langle\chi_{0}^{v}\right|\right]\left(\mathbf{I} \otimes \boldsymbol{\Lambda}_{01}\right)\left(\left|\Phi_{+}\right\rangle_{S^{\prime}}\left\langle\Phi_{+}\right|\right)\right\} .
$$

Maximization in equation (28) can be extended to all operators $\hat{U}_{S S^{\prime}}$ satisfying $\hat{U}_{S S^{\prime}}^{\dagger} \hat{U}_{S S^{\prime}} \leq \hat{\mathbb{1}}$. Therefore, for any such operator we have

$$
V_{G} \geq d\left|\operatorname{Tr}_{S S^{\prime}}\left(\left(\sqrt{\left(\hat{\varrho}_{1}\right)_{S}^{T}} \hat{U}_{S S^{\prime}} \sqrt{\left(\hat{\varrho}_{0}\right)_{S}^{T}}\right)\left(\mathbf{I} \otimes \boldsymbol{\Lambda}_{01}\right)\left(\left|\Phi_{+}\right\rangle_{S S^{\prime}}\left\langle\Phi_{+}\right|\right)\right)\right|,
$$

where we used equation (30). Consequently, tracing both sides of equation (14) multiplied by $d\left(\mathbf{I} \otimes \boldsymbol{\Lambda}_{01}\right)\left(\left|\Phi_{+}\right\rangle\left\langle\Phi_{+}\right|\right)$and comparing resulting expressions with equations (31) and (32) immediately yields a lower bound on the generalized visibility presented in equation (15).

\section{References}

1. Bohr, N. Das Quantenpostulat und die neuere Entwicklung der Atomistik. Naturwissenschaften 16, 245-257 (1928).

2. Bohr, N. The quantum postulate and the recent development of atomic theory. Nature 121, 580-590 (1928). 
3. Wootters, W. K. \& Zurek, W. H. Complementarity in the double-slit experiment: quantum nonseparability and a quantitative statement of Bohr's principle. Phys. Rev. D 19, 473-484 (1979).

4. Scully, M. O., Englert, B.-G. \& Walther, H. Quantum optical tests of complementarity. Nature 351, 111-116 (1991).

5. Greenberger, D. M. \& Yasin, A. Simultaneous wave and particle knowledge in a neutron interferometer. Phys. Lett. A 128, 391-394 (1988).

6. Englert, B.-G. Fringe visibility and which-way information: an inequality. Phys. Rev. Lett. 77, 2154-2157 (1996).

7. Jaeger, G., Shimony, A. \& Vaidman, L. Two interferometric complementarities. Phys. Rev. A 51, 54-67 (1995).

8. Björk, G. \& Karlsson, A. Complementarity and quantum erasure in welcher Weg experiments. Phys. Rev. A 58, 3477-3483 (1998).

9. Jacques, V. et al. Experimental realization of Wheeler's delayed-choice gedanken experiment. Science 315, 966-968 (2007).

10. Peruzzo, A., Shadbolt, P., Brunner, N., Popescu, S. \& O’Brien, J. L. A quantum delayed-choice experiment. Science 338, 634-637 (2012).

11. Kaiser, F., Coudreau, T., Milman, P., Ostrowsky, D. B. \& Tanzilli, S. Entanglement-enabled delayed-choice experiment. Science 338, 637-640 (2012).

12. Helstrom, C. W. Quantum Detection and Estimation Theory (Academic Press, 1976).

13. Chuang, I. L. \& Nielsen, M. A. Prescription for experimental determination of the dynamics of a quantum black box. J. Mod. Opt. 44, 2455-2467 (1997).

14. Poyatos, J. F., Cirac, J. I. \& Zoller, P. Complete characterization of a quantum process: the two-bit quantum gate. Phys. Rev. Lett. 78, 390-393 (1997).

15. D’Ariano, G. M. \& Lo Presti, P. Quantum tomography for measuring experimentally the matrix elements of an arbitrary quantum operation. Phys. Rev. Lett. 86, 4195-4198 (2001).

16. Fuchs, C. A. \& van de Graaf, J. Cryptographic distinguishability measures for quantum-mechanical states. IEEE Trans. Inf. Theory 45, 1216-1227 (1999).

17. Nielsen, M. A. \& Chuang, I. L. Quantum Computation and Quantum Information (Cambridge University Press, Cambridge, 2000).

18. Uhlmann, A. The 'transition probability' in the state space of $\mathrm{a}^{*}$-algebra. Rep. Math. Phys. 9, 273-279 (1976).

19. Jozsa, R. Fidelity for mixed quantum states. J. Mod. Opt. 41, 2315-2323 (1994).

20. Scully, M. O. \& Drühl, K. Quantum eraser: a proposed photon correlation experiment concerning observation and 'delayed choice' in quantum mechanics. Phys. Rev. A 25, 2208-2213 (1982).

21. Englert, B.-G. \& Bergou, J. A. Quantitative quantum erasure. Opt. Comm. 179, 337-355 (2000)

22. Kim, Y.-H., Yu, R., Kulik, S. P. \& Shih, Y. Delayed 'choice' quantum eraser. Phys. Rev. Lett. 84, 1-5 (2000).

23. Ma, X.-S. et al. Quantum erasure with causally disconnected choice. Proc. Natl Acad. Sci. 110, 1221-1226 (2013).
24. Horodecki, K., Horodecki, M., Horodecki, P. \& Oppenheim, J. Secure key from bound entanglement. Phys. Rev. Lett. 94, 160502 (2005).

25. Horodecki, K., Horodecki, M., Horodecki, P. \& Oppenheim, J. General paradigm for distilling classical key from quantum states. IEEE Trans. Inf. Theory 55, 1898-1929 (2009).

26. Dobek, K., Karpiński, M., Demkowicz-Dobrzański, R., Banaszek, K. \& Horodecki, P. Experimental extraction of secure correlations from a noisy private state. Phys. Rev. Lett. 106, 030501 (2011).

27. Ozols, M., Smith, G. \& Smolin, J. A. Bound entangled states with secret key and their classical counterpart. Preprint at http://arxiv.org/abs/1305.0848 (2013).

28. Banaszek, K., Horodecki, K. \& Horodecki, P. Quantum privacy witness. Phys Rev. A 85, 012330 (2012)

29. Choi, M.-D. Positive linear maps on $C^{\star}$-algebras. Can. J. Math. 24, 520-529 (1972).

30. Jamiołkowski, A. Linear transformations which preserve trace and positive semidefiniteness of operators. Rep. Math. Phys. 3, 275-278 (1972).

\section{Acknowledgements}

We wish to acknowledge insightful discussions with B.-G. Englert, P. Raynal, R. Demkowicz-Dobrzański and W. Wasilewski. This research was supported by the European Union FP7 project Q-ESSENCE (Grant Agreement no. 248095), ERA-NET project QUASAR, and the Foundation for Polish Science TEAM project cofinanced by the EU European Regional Development Fund. Part of the work was done in National Quantum Information Centre of Gdańsk.

\section{Author contributions}

All authors contributed extensively to the work presented in this paper.

\section{Additional information}

Competing financial interests: The authors declare no competing financial interests.

Reprints and permission information is available online at http://npg.nature.com/ reprintsandpermissions/

How to cite this article: Banaszek, K. et al. Quantum mechanical which-way experiment with an internal degree of freedom. Nat. Commun. 4:2594 doi: 10.1038/ncomms3594 (2013).

(1) This work is licensed under a Creative Commons Attributionthis license, visit http://creativecommons.org/licenses/by-nc-sa/3.0/ 